\title{
Topological properties of the SU(3) random vortex world-surface model
}

\author{
Michael Engelhardt* $*$ \\ Department of Physics, New Mexico State University, Las Cruces, NM 88003, USA \\ E-mail: engel @nmsu. edu
}

\begin{abstract}
The random vortex world-surface model is an infrared effective model of Yang-Mills dynamics based on center vortex degrees of freedom. These degrees of freedom carry topological charge through writhe and self-intersection of their world-surfaces. A practical implementation of the model realizes the vortex world-surfaces by composing them of elementary squares on a hypercubic lattice. The topological charge for specifically such configurations is constructed in the case of SU(3) color. This necessitates a proper treatment of vortex color structure at vortex branchings, a feature which is absent in the SU(2) color case investigated previously. On the basis of the construction, the topological susceptibility is evaluated in the random vortex world-surface ensemble, both in the confined low-temperature as well as in the deconfined high-temperature phase.
\end{abstract}

8th Conference Quark Confinement and the Hadron Spectrum September 1-6, 2008

Mainz. Germany

\footnotetext{
* Speaker.

${ }^{\dagger}$ Supported by the U.S. DOE under grant DE-FG02-96ER40965.
} 


\section{Introduction}

The random vortex world-surface model [1-8] is an infrared effective description of the strong interaction vacuum which starts from the assumption that the relevant gluonic degrees of freedom are center vortices. These are tubes of chromomagnetic flux which are envisioned to be percolating throughout space. The flux tubes are closed, since chromomagnetic flux must be continuous in accordance with the Bianchi constraint, and their flux is quantized in a way which is determined by the center of the gauge group. Adopting a four-dimensional (Euclidean) space-time point of view, an ensemble of random vortex world-surfaces is constructed (in practice, employing Monte-Carlo methods), and relevant observables are then calculated within this ensemble.

The physical picture of the strong interaction vacuum sketched above, motivating the random vortex world-surface model, was originally proposed as an explanation of the confinement phenomenon $[9,10]$. The percolating random distribution of magnetic flux sufficiently disorders Wilson loops such as to generate an area law for them. In the course of subsequent investigations [11-18] sparked by the development of techniques which permit the detection and study of center vortices in lattice gauge configurations $[12,14]$, a more comprehensive picture emerged. Not only does the vortex vacuum generate confinement, but it also can account for the other two core characteristics of the strong interaction, namely, the spontaneous breaking of chiral symmetry, and the axial $U_{A}(1)$ anomaly.

The present investigation further contributes to this broadening of the scope of the vortex picture. While the confinement properties of the random vortex world-surface model have been investigated in a variety of settings [1,4-8], the topological and chiral properties had hitherto only been studied for the simplest case of $S U(2)$ color [2,3]. Here, the topological properties are investigated for $S U(3)$ color. After dealing with some new subtleties concerning vortex color structure which are not present in the $S U(2)$ case, the topological susceptibility of the $S U(3)$ random vortex world-surface ensemble is evaluated as a function of temperature, including both the confined as well as the deconfined phase.

\section{Color structure of $\mathrm{SU}(3)$ center vortices and topological charge}

The chromomagnetic flux carried by a center vortex is measured by Wilson loops $W_{C}$ encircling the vortex along paths $C$. Vortex flux quantization expresses itself in the fact that such Wilson loops take only (nontrivial) values in the center of the gauge group, for $S U(3)$,

$$
W_{C}=\frac{1}{3} \operatorname{Tr} \mathscr{P} \exp \left(i \oint_{C} A_{\mu} d x_{\mu}\right)=\exp ( \pm 2 \pi i / 3) .
$$

Since the two center elements in (2.1) are related by complex conjugation, the corresponding vortex fluxes are related by inversion of their space-time orientation. There is, therefore, only one type of vortex in the $S U$ (3) case. Nevertheless, vortex flux can branch; the Bianchi constraint admits the possibility of a flux associated with the center phase $\exp (-2 \pi i / 3)=\exp (4 \pi i / 3)$ splitting into two oppositely oriented fluxes, each associated with the center phase $\exp (2 \pi i / 3)$.

Evaluating the topological charge

$$
Q=\frac{1}{32 \pi^{2}} \int d^{4} x \varepsilon_{\mu \nu \lambda \tau} \operatorname{Tr} F_{\mu \nu} F_{\lambda \tau}
$$


calls for a representation of vortex flux via the algebra of the gauge group. This is conveniently achieved by adopting an Abelian gauge, in which the gauge field $A_{\mu}$ and its field strength $F_{\mu \nu}$ are proportional to diagonal matrices $T$ in color space. Contrary to the $S U(2)$ case, in which there is no residual (gauge) freedom in the set of matrices $T$ entering the description, for $S U(3)$, there is such a choice. In order to generate both nontrivial center elements when evaluating Wilson loops,

$$
W=(1 / 3) \operatorname{Tr} \exp (2 \pi i T / 3)=\exp ( \pm 2 \pi i / 3),
$$

one minimally needs a set with two elements, e.g., $T \in\{ \pm \operatorname{diag}(1,1,-2)\}$. However, such a choice has the drawback that vortex branchings necessarily carry Abelian magnetic monopoles (why this is a drawback will be explained further below): If a vortex associated with the center element $\exp (-2 \pi i / 3)$ splits into two oppositely oriented vortices, flux is only continuous modulo $2 \pi$ in each color component,

$$
\frac{2 \pi}{3} \operatorname{diag}(-1,-1,2)=2 \cdot \frac{2 \pi}{3} \operatorname{diag}(1,1,-2)-2 \pi \operatorname{diag}(1,1,-2) .
$$

In order to always be able to deform Abelian magnetic monopole world-lines ${ }^{1}$ away from vortex world-surface branchings, a more flexible basis is necessary [19], such as

$$
T \in\{ \pm \operatorname{diag}(1,1,-2), \pm \operatorname{diag}(1,-2,1), \pm \operatorname{diag}(-2,1,1)\} .
$$

A description of vortex surfaces in terms of patches which are each associated with one of the elements in (2.5) is well-suited for the evaluation of topological charge.

According to (2.2), topological charge density is generated when field strength components $F_{\mu \nu}, F_{\lambda \tau}$ exist at a given space-time position such that $\mu, v, \lambda, \tau$ span all four space-time dimensions. Since a vortex world-surface is associated with a field strength component $F_{\mu \nu}$ such that $\mu, v$ denote the directions perpendicular to the world-surface ${ }^{2}$, this translates into the statement that the vortex world-surfaces present at a given space-time position must span all four space-time dimensions. There are two ways in which this can take place, namely, if two distinct surfaces intersect, or if one surface writhes such that it explores all four directions [2,19-22].

To have a practicable scheme of generating an ensemble of random vortex world-surfaces, the latter are composed of elementary squares on a hypercubic lattice. In such a setting, topological charge density only occurs at lattice sites. This simplified description comes, however, at a price. Due to the discrete set of directions in which surfaces can extend, ambiguous features appear in the configurations, features which would merely constitute a negligible set of measure zero if one considered arbitrary surfaces in continuous space-time. For one, two surfaces may coincide along whole line segments in space-time instead of only discrete intersection points. In this case, there is generally no unambiguous assignment of the participating vortex elementary squares to two distinct surfaces. To remedy this, vortex surfaces are transferred to a finer lattice and slightly deformed (by less than half the original lattice spacing) until all ambiguities are removed. While this permits the assignment of a topological charge, the described deformation procedure can introduce additional

\footnotetext{
${ }^{1}$ Note that magnetic monopole world-lines generally cannot be eliminated altogether due to the nonorientability of generic world-surfaces.

${ }^{2}$ E.g., a static chromomagnetic flux in 3-direction, which corresponds to a world-surface extending in the 3- and 4-directions, carries a 3 -component of the magnetic field, $B_{3}$, and thus a field strength component $F_{12}$.
} 


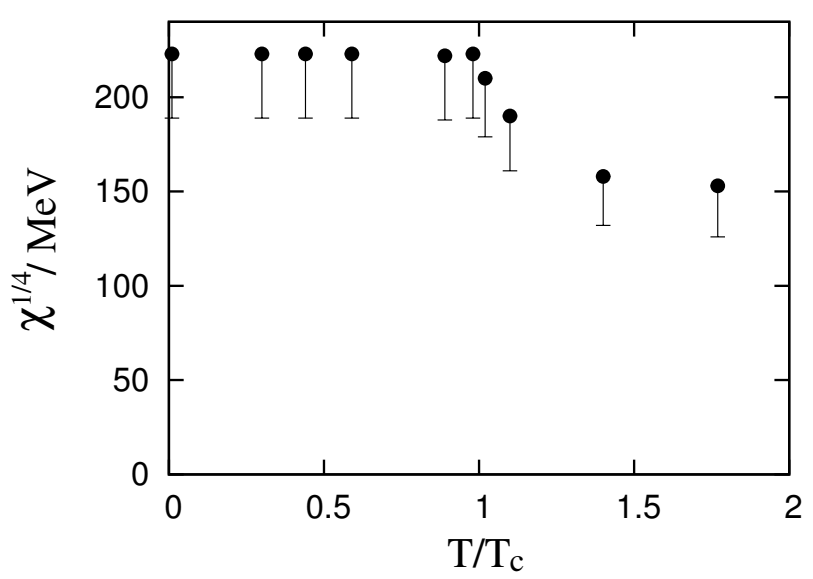

Figure 1: Fourth root of the topological susceptibility measured in the $S U(3)$ random vortex world-surface model, as a function of temperature. In the employed random surface ensemble, residual statistical uncertainties are smaller than the filled circle symbols depicting the measured data; the origin of the displayed systematic downward uncertainty is explained in the main text.

spurious topological charge density at the fine lattice scale, leading to an overestimate of the topological susceptibility. This in fact constitutes the main source of error in the measurement. The corresponding downward uncertainty in the data shown in Fig. 1 is estimated by rescaling with the (appropriate power of the) vortex density, which also is increased by the deformation procedure in a way which is expected to roughly track the spurious increase in the topological charge density.

A further ambiguity present in the configurations is related to Abelian magnetic monopole world-lines. If tied rigidly to vortex branchings, they necessarily run through lattice sites, precisely where topological charge density is concentrated. Such singular coincidences also preclude an unambiguous assignment of topological charge. They are removed by deforming monopole worldlines away from lattice sites, taking advantage of the flexible color structure defined by (2.5).

\section{Random vortex world-surface model}

As already mentioned above, and described in detail in [1,4], a practical Monte-Carlo scheme for generating a random vortex world-surface ensemble is arrived at by composing the surfaces from elementary squares on a hypercubic lattice. The lattice spacing is a fixed physical quantity related to the vortex thickness, which defines the ultraviolet cutoff of the model; it amounts to $0.39 \mathrm{fm}$ if one sets the scale via the zero-temperature string tension, $\sigma=(440 \mathrm{MeV})^{2}$. The ensemble is weighted by an action penalizing vortex world-surface curvature,

$$
S=c \times \square
$$

i.e., each adjacent, non-coplanar pair of vortex squares adds an increment $c$ to the action. The coefficient $c$ is tuned to reproduce the value $T_{c} / \sqrt{\sigma}=0.63$ obtained in $S U$ (3) lattice Yang-Mills theory, where $T_{c}$ denotes the deconfinement temperature ${ }^{3}$. This yields $c=0.21$. Evaluating the topological charge $Q$ of configurations as indicated in the previous section yields the result shown in Fig. 1 for the topological susceptibility $\chi=\left\langle Q^{2}\right\rangle / V$ (where $V$ denotes the lattice four-volume).

\footnotetext{
${ }^{3}$ The temperature in the model is varied as usual, via the Euclidean time extent of the lattice.
} 


\section{Conclusions}

The raw data depicted in Fig. 1 lie somewhat above analogous results obtained in $S U(3)$ lattice Yang-Mills theory. The latter are concentrated around $\chi^{1 / 4} \approx 190 \mathrm{MeV}$, although there is a considerable spread in reported values, cf. in particular Table 1 in [23]. For finite temperatures, cf., e.g., [24]. However, as indicated in section 2, ambiguities present in the vortex configurations due to the specific hypercubic realization of their world-surfaces employed in this work leave a substantial systematic downward uncertainty in the results, as shown in Fig. 1. Taking this uncertainty into consideration, it cannot be excluded that the $S U(3)$ random vortex world-surface ensemble possesses a topological susceptibility compatible with the $S U$ (3) Yang-Mills one. To resolve this uncertainty, a more flexible construction of the world-surfaces, allowing them to extend into arbitrary directions in space-time, is needed, e.g., a construction in terms of random triangulations.

\section{References}

[1] M. Engelhardt and H. Reinhardt, Nucl. Phys. B585 (2000) 591.

[2] M. Engelhardt, Nucl. Phys. B585 (2000) 614.

[3] M. Engelhardt, Nucl. Phys. B638 (2002) 81.

[4] M. Engelhardt, M. Quandt and H. Reinhardt, Nucl. Phys. B685 (2004) 227.

[5] M. Engelhardt, Phys. Rev. D 70 (2004) 074004.

[6] M. Quandt, H. Reinhardt and M. Engelhardt, Phys. Rev. D 71 (2005) 054026.

[7] M. Engelhardt, Phys. Rev. D 73 (2006) 034015.

[8] M. Engelhardt and B. Sperisen, Phys. Rev. D 74 (2006) 125011.

[9] G. 't Hooft, Nucl. Phys. B138 (1978) 1.

[10] Y. Aharonov, A. Casher and S. Yankielowicz, Nucl. Phys. B146 (1978) 256.

[11] J. Greensite, Prog. Part. Nucl. Phys. 51 (2003) 1.

[12] L. Del Debbio, M. Faber, J. Giedt, J. Greensite and Š. Olejník, Phys. Rev. D 58 (1998) 094501.

[13] P. de Forcrand and M. D’Elia, Phys. Rev. Lett. 82 (1999) 4582.

[14] C. Alexandrou, M. D'Elia and P. de Forcrand, Nucl. Phys. Proc. Suppl. 83 (2000) 437.

[15] M. Engelhardt, K. Langfeld, H. Reinhardt and O. Tennert, Phys. Lett. B431 (1998) 141.

[16] M. Engelhardt, K. Langfeld, H. Reinhardt and O. Tennert, Phys. Rev. D 61 (2000) 054504.

[17] R. Bertle, M. Engelhardt and M. Faber, Phys. Rev. D 64 (2001) 074504.

[18] R. Höllwieser, M. Faber, J. Greensite, U. M. Heller and Š. Olejník, arXiv:0805.1846 [hep-lat].

[19] J. M. Cornwall, Phys. Rev. D 58 (1998) 105028.

[20] M. Engelhardt and H. Reinhardt, Nucl. Phys. B567 (2000) 249.

[21] F. Bruckmann and M. Engelhardt, Phys. Rev. D 68 (2003) 105011.

[22] G. Jordan, R. Höllwieser, M. Faber and U. M. Heller, Phys. Rev. D 77 (2008) 014515.

[23] E. Vicari and H. Panagopoulos, arXiv:0803.1593 [hep-th].

[24] B. Allés, M. D’Elia and A. Di Giacomo, Nucl. Phys. B494 (1997) 281; erratum ibid. B679 (2004) 397. 